\title{
An Ideal Convergence
}
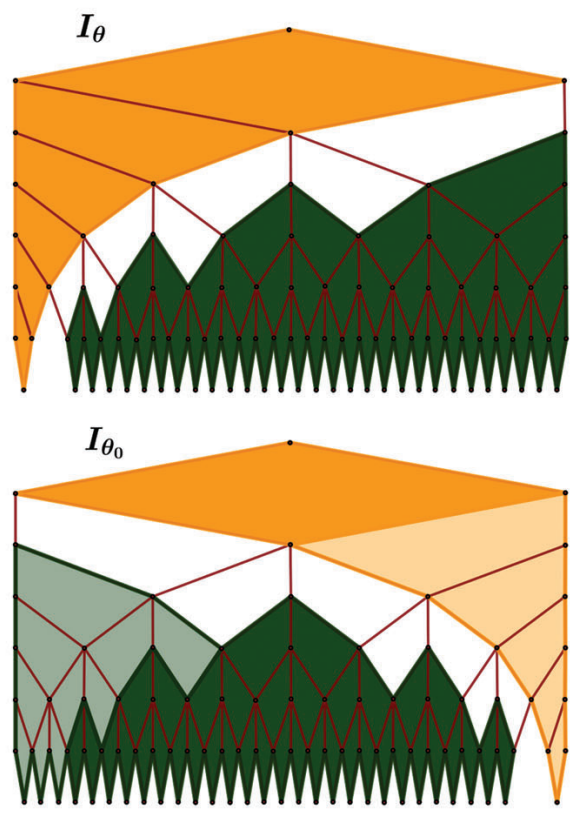
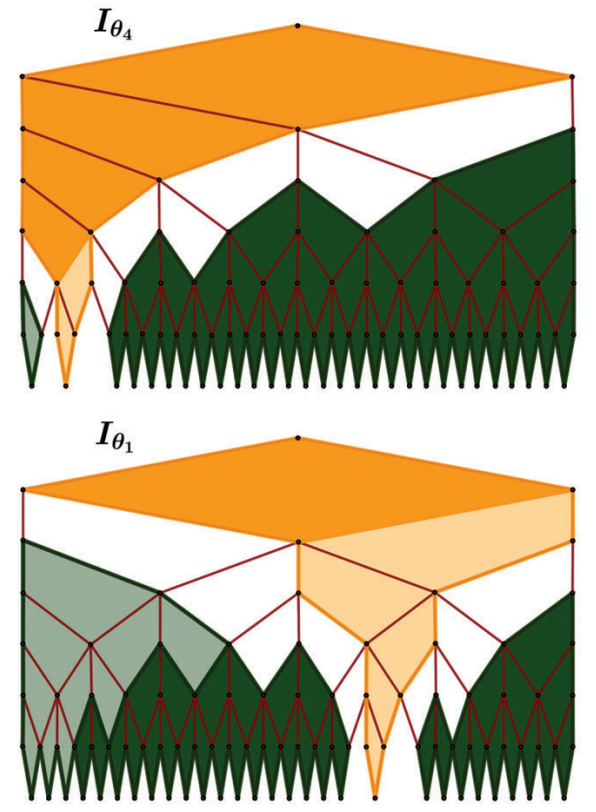
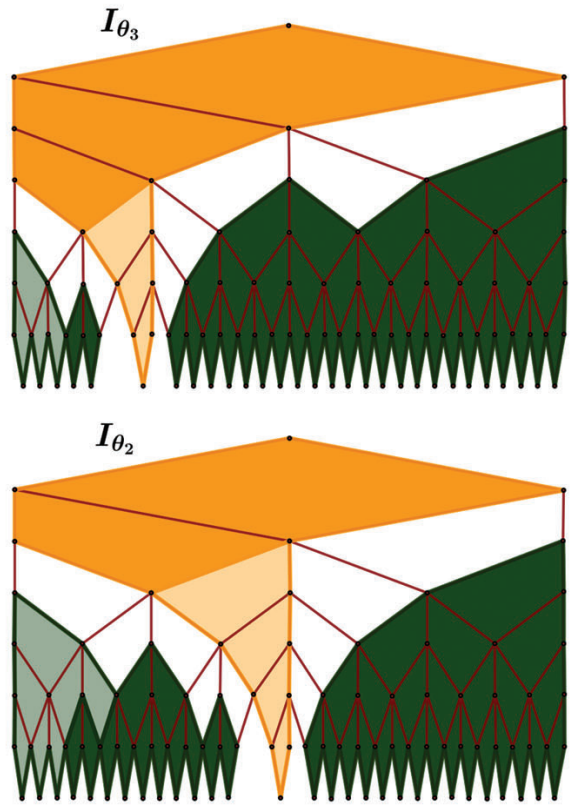

\section{Konrad Aguilar, Samantha Brooker, Frédéric Latrémolière, and Alejandra López}

\section{Introduction}

Quantum physics describes the world using noncommuting variables modeled by operators on a complex Hilbert space. The study of algebras of bounded operators on Hilbert spaces began with the work of J. von Neumann on the foundations of quantum mechanics, and soon found other areas of applications. In particular, I. M. Gelfand introduced $\mathrm{C}^{*}$-algebras, which are, up to an appropriate notion of *-isomorphism, certain algebras of bounded operators on a Hilbert space. These algebras include as important examples: the algebra of $n \times n$-matrices over $\mathbb{C}$

Konrad Aguilar is an assistant professor at Pomona College. His email address is konrad. agui 1 ar@pomona. edu.

Samantha Brooker is a graduate student at Arizona State University. Her email address is sjbrooke@asu. edu.

Frédéric Latrémolière is a professor at the University of Denver. His email address is frederic.1atremo1iere@du.edu.

Alejandra López is a graduate student at Purdue University. Her email address is 1opez408@purdue.edu.

Communicated by Notices Associate Editor Stephan Ramon Garcia.

For permission to reprint this article, please contact:

reprint-permission@ams.org.

DOI: https://doi.org/10.1090/noti2338 (denoted $M_{n}(\mathbb{C})$ ), and the algebra of $\mathbb{C}$-valued continuous functions over a compact Hausdorff space $X$ (denoted $C(X)$ ) with the supremum norm and pointwise conjugation for the adjoint operation. Moreover, $\mathrm{C}^{*}$-algebras naturally appear in representation theory, dynamics, and geometry, in addition to physics [Dav96].

We will give a more detailed description of $\mathrm{C}^{*}$-algebras later, but for now, we note that any unital commutative $\mathrm{C}^{*}$-algebra is (up to ${ }^{*}$-isomorphism) $C(X)$ for some compact Hausdorff space $X$. This fact is part of Gelfand's celebrated contravariant duality between the category of unital commutative $\mathrm{C}^{*}$-algebras and the category of compact Hausdorff spaces, which sparked a new area of research in functional and geometric analysis focused on developing the analogues of various topological and geometric concepts for noncommutative $\mathrm{C}^{*}$-algebras [Dav96]. A. Connes' noncommutative geometry is a prime example of this development, and, in particular, Connes opened the path for the study of quantum analogues of compact metric spaces [Con94]. The theory of compact quantum metric spaces began in earnest with the work of M. A. Rieffel [Rie98], with a focus on constructing a topology on the class of compact quantum metric spaces to provide a formal framework for approximations of classical and 
quantum spaces found in the mathematical physics literature [Rie04].

This formal framework begins with tools from classical metric geometry. Indeed, given a metric space $(X, d)$, the Hausdorff distance provides a way to approximate subsets by other subsets, and is defined for any two closed and bounded subsets $A$ and $B$ of $X$ by

$$
\text { Haus }_{d}(A, B)=\max \left\{\sup _{x \in A} d(x, B), \sup _{x \in B} d(x, A)\right\},
$$

where $d(x, C)=\inf _{y \in C} d(x, y)$ for any subset $C$ of $X$. For instance, the circle $\mathbb{T}$ can be approximated by the groups of $n$th roots of unity $\mathbb{U}_{n}=\{\exp (2 \pi i k / n) \mid k=0, \ldots, n-1\}$ for the Hausdorff distance induced by the Euclidean distance on the plane $d_{\|\cdot\|_{2}}$ (see Figure 1). Furthermore, this
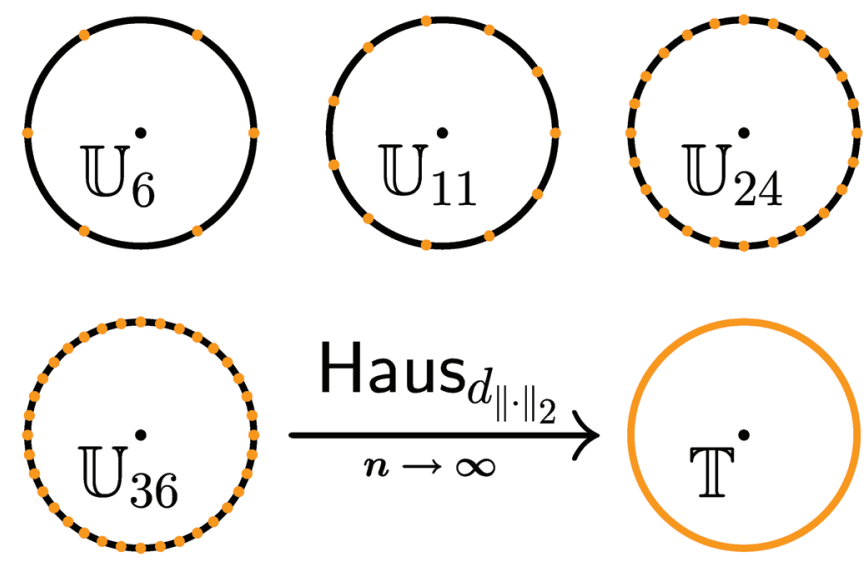

Figure 1. Convergence of $\mathbb{U}_{n}$ to $\mathbb{T}$.

example of Hausdorff convergence provides motivation for an important example coming from physics that we will address later (see the discussion at equation (1)).

Rieffel used the Hausdorff distance to develop a generalization of the Gromov-Hausdorff distance [Gro81] to noncommutative analogues of compact metric spaces, in order to formalize statements from the high-energy physics literature about approximations of (possibly quantum) spaces by matrix algebras [Rie04], which spawned a new area of study called noncommutative metric geometry. This article aims to provide a glimpse of this area by introducing some of the main structures involved and examples stemming from physics and $\mathrm{C}^{*}$-algebra theory. In fact, the opening graphic comes from an intriguing connection between noncommutative metric geometry and $\mathrm{C}^{*}$-algebra theory with a focus on ideals of $\mathrm{C}^{*}$-algebras (which we can represent with graphs for certain classes of $\mathrm{C}^{*}$-algebras) and a notion of convergence of ideals. Moreover, the graphs of the ideals of the particular example we focus on (the Boca-Mundici algebra [Boc08]) are given by the continued fraction expansions of irrational numbers. Continued fractions have been used several other times in the study of $\mathrm{C}^{*}$-algebras to build some fascinating structures and prove some spectacular results (see the construction of the Effros-Shen algebras in [Dav96, Section VI.3] and the proof of the "main step" in the classification of a fundamental class of $\mathrm{C}^{*}$-algebras in [EE93]), which are related to the structures we present in this paper. To end this article, we list some future work motivated, in part, by the work we highlighted in this article.

We also note that, although we mainly present examples of noncommutative spaces, throughout the paper we visit the commutative case whenever a new concept is discussed. This serves two purposes. First, it allows us to provide a connection to classical structures such as the Cantor set (see Figure 2 and Theorems 3.6 and 3.7). Second, we use the commutative case as a more familiar setting to describe the intuition for some of the approaches in the noncommutative setting (see Theorems 3.12 and 3.13 for connections to convergence of ideals and the Hausdorff distance).

\section{Compact Quantum Metric Spaces and the Gromov-Hausdorff Propinquity}

To begin our journey of defining the core objects of noncommutative metric geometry, we must begin with $\mathrm{C}^{*}$ algebras. We started this article with bounded operators on a Hilbert space. Therefore, once we define these terms, we move onto a useful and fundamental abstract definition of $\mathrm{C}^{*}$-algebras due to Gelfand, M. Naimark, and I. Segal. From this, we are then in a position to discuss further structures related to $\mathrm{C}^{*}$-algebras (e.g., states) that allow us to tie the theory of metric spaces to the study of $\mathrm{C}^{*}$-algebras by way of commutative $\mathrm{C}^{*}$-algebras. This then gives us the foundation we need to define the notion of a noncommutative/quantum metric space and the tools to generalize the Gromov-Hausdorff distance, while also describing how this provides a framework to answer questions about approximations of spaces by matrix algebras. All the facts we present about $\mathrm{C}^{*}$-algebras can be found in [Dav96].

Thus, we begin this journey with: a Hilbert space $H$ is a vector space (which we always take over the field $\mathbb{C}$ of complex numbers) which is complete for the norm induced by an inner product $\langle\cdot, \cdot\rangle_{H}$. An inner product $\langle\cdot, \cdot\rangle_{H}: H \times H \rightarrow \mathbb{C}$ on a vector space $H$ is a function such that:

1. $\forall \xi \in H \quad\langle\xi, \xi\rangle_{H} \geq 0$,

2. $\forall \xi \in H \quad\langle\xi, \xi\rangle_{H}=0 \Longleftrightarrow \xi=0$,

3. $\forall \xi \in H \quad \eta \in H \mapsto\langle\eta, \xi\rangle_{H}$ is linear,

4. $\forall \xi, \eta \in H \quad\langle\xi, \eta\rangle_{H}=\overline{\langle\eta, \xi\rangle_{H}}$.

An inner product $\langle\cdot, \cdot\rangle_{H}$ on a vector space $H$ defines a norm by setting $\|\xi\|_{H}=\sqrt{\langle\xi, \xi\rangle_{H}}$ for all $\xi \in H$. An example of a Hilbert space is given by $\mathbb{C}^{n}$ for its usual inner product. An infinite-dimensional example of a Hilbert space is given 
by $\ell^{2}(\mathbb{N})=\left\{\left(\xi_{n}\right)_{n \in \mathbb{N}} \in \mathbb{C}^{\mathbb{N}}: \sum_{n \in \mathbb{N}}\left|x_{n}\right|^{2}<\infty\right\}$ for the inner product defined by $\left\langle\left(\xi_{n}\right)_{n \in \mathbb{N}},\left(\eta_{n}\right)_{n \in \mathbb{N}}\right\rangle_{\ell^{2}(\mathbb{N})}=\sum_{n \in \mathbb{N}} \xi_{n} \overline{\eta_{n}}$.

A bounded operator on a Hilbert space $H$ is a continuous linear map from $H$ to itself. The term bounded refers to the property that a linear map on any normed vector space is continuous if and only if it is bounded on the set of vectors of norm 1. In particular, for any continuous linear map $a: H \rightarrow H$, we define the operator norm $\|a\|_{B(H)}$ of $a$ as $\sup \left\{\|a(\xi)\|_{H}: \xi \in H,\|\xi\|_{H} \leq 1\right\}$.

As the composition of bounded operators on a Hilbert space $H$ is again a bounded operator on $H$, the space $B(H)$ of all bounded operators on a Hilbert space is an associative algebra. There is one additional operation on $B(H)$, called the adjoint, and denoted by a superscript $*$, which is uniquely defined for any $a \in B(H)$ by the relation

$$
\forall \xi, \eta \in H \quad\left\langle a^{*}(\xi), \eta\right\rangle_{H}=\langle\xi, a(\eta)\rangle_{H} .
$$

The resulting structure on $B(H)$ is an example of a $\mathrm{C}^{*}$ algebra, a type of operator algebra introduced by Gelfand. Indeed, we can abstract the properties of $B(H)$ as follows, which we present now along with subobjects and morphisms.

Definition 2.1. A Banach algebra $A$ is a normed algebra with norm $\|\cdot\|_{A}$ that is complete with respect to the norminduced metric, and such that $\|a b\|_{A} \leq\|a\|_{A} \cdot\|b\|_{A}$ for all $a, b \in A$.

Definition 2.2. A $C^{*}$-algebra $A$ is a Banach algebra equipped with a map ${ }^{*}: A \rightarrow A$ called the adjoint satisfying for all $a, b \in A, \mu \in \mathbb{C}$ :

1. $\left(a^{*}\right)^{*}=a,(a b)^{*}=b^{*} a^{*},(a+\mu b)^{*}=a^{*}+\bar{\mu} b^{*}$,

2. (C*-identity) $\left\|a a^{*}\right\|_{A}=\|a\|_{A}^{2}$.

We say that $A$ is unital if it has a multiplicative identity, $1_{A}$. A subalgebra $B \subseteq A$ is a $C^{*}$-subalgebra if it is norm-closed and self-adjoint $\left(b \in B \Longleftrightarrow b^{*} \in B\right)$.

If $B$ is a $C^{*}$-algebra, then $\pi: A \rightarrow B$ is a ${ }^{*}$-morphism if it is an adjoint-preserving multiplicative linear map, and is a ${ }^{*}$-isomorphism if it is a bijective *-morphism. We say $A$ is ${ }^{*}$-isomorphic to $B$ if there exists a *-isomorphism between $A$ and $B$ and denote this by $A \cong B$.

Gelfand proved that $A$ is a $\mathrm{C}^{*}$-algebra if and only if there exists a Hilbert space $H$, and an injective ${ }^{*}$-morphism from $A$ into $B(H)$. Notably, ${ }^{*}$-morphisms are continuous; moreover injective ${ }^{*}$-morphisms are isometric. So every $\mathrm{C}^{*}$ algebra is ${ }^{*}$-isomorphic to a norm-closed, adjoint-closed algebra of bounded operators on a Hilbert space.

Given a compact Hausdorff space $X$, the algebra of $\mathbb{C}$ valued continuous functions over $X$, with the usual pointwise addition and multiplication operation, the supremum norm $f \in C(X) \mapsto\|f\|_{C(X)}=\sup _{x \in X}|f(x)|$, and the adjoint given by pointwise conjugation, is a unital commutative $\mathrm{C}^{*}$-algebra. Remarkably, Gelfand proved that any unital commutative $\mathrm{C}^{*}$-algebra is *-isomorphic to the $\mathrm{C}^{*}$ algebra $C(X)$ for some compact Hausdorff space, which is uniquely determined by $A$, up to a homeomorphism.

This correspondence between unital commutative $\mathrm{C}^{*}$ algebras and compact Hausdorff spaces can actually be understood as a duality of categories, between the category of unital commutative $\mathrm{C}^{*}$-algebras and their ${ }^{*}$-morphisms, and the category of compact Hausdorff spaces and their continuous functions. For our purpose, it is interesting to give a description of this correspondence at the level of the objects.

An element $a$ of a $\mathrm{C}^{*}$-algebra $A$ is self-adjoint when $a=$ $a^{*}$. Since, for any element $a \in A$, we have $a=\frac{a+a^{*}}{2}+i \frac{a-a^{*}}{2 i}$, we see that the self-adjoint elements of $A \operatorname{span} A$. The space $s a(A)$ of self-adjoint elements of a $\mathrm{C}^{*}$-algebra is an ordered vector space, where we define a positive element $a \in A$ as any element of the form $b^{*} b$ for some $b \in A$. This order generalizes the order on Hermitian matrices. With this order, we can define a class of positive linear functionals over a C*-algebra. Specifically, a state of a unital $\mathrm{C}^{*}$-algebra $A$ is a linear functional from $A$ to $\mathbb{C}$ such that if $a \in \operatorname{sa}(A)$ and $a$ is positive, then $\varphi(a) \geq 0$, and $\varphi$ maps the unit of $A$ to 1 . A state of a $\mathrm{C}^{*}$-algebra is always continuous, and thus an element of the dual (Banach space) of $A$. The normalized trace on $M_{n}(\mathbb{C})\left(a \in M_{n}(\mathbb{C}) \mapsto \frac{1}{n} \operatorname{Tr}(a)\right)$ is a standard example of a state. The state space $S(A)$ of $A$ is the set of all states over $A$. It is a convex, weak*-compact subset of the dual of $A$.

Among all the states of a unital $\mathrm{C}^{*}$-algebra $A$ are the characters of $A$, which are unit-preserving *-morphisms from $A$ to $\mathbb{C}$. However, for a general $\mathrm{C}^{*}$-algebra $A$, there may be no characters at all! When $A$ is a unital commutative $\mathrm{C}^{*}$-algebra, however, the subset $G$ of all the characters of $A$ is a weak*-closed subset of $S(A)$-hence, a compact Hausdorff space-such that $A$ is indeed *-isomorphic to $C(G)$. With this identification, by the Radon-Riesz Theorem, the state space $S(A)$ is the space of integrals against Radon probability measures over $G$. Thus, characters of a unital $\mathrm{C}^{*}$-algebra can be seen as (a generalization of) points.

When working with a general (i.e., not necessarily commutative) unital $\mathrm{C}^{*}$-algebra $A$, there may be no character, hence no point, but there still are states. In fact, the state space of a unital $\mathrm{C}^{*}$-algebra spans the dual of the $\mathrm{C}^{*}$ algebra. Thus, while we lose the notion of a point, we keep the notion of a "probability." This structure is a simple example of a deeper idea. Indeed, the study of $\mathrm{C}^{*}$-algebras, in the noncommutative geometry of Connes and the noncommutative metric geometry of Rieffel, provides noncommutative analogues of classical analytical structures to answer various profound questions. 
To introduce the subject of noncommutative metric geometry, we begin with a common special case of the general problem in physics to find approximations of infinitedimensional problems by finite-dimensional ones. The clock and shift matrices are a standard example from quantum dynamics for finite-dimensional systems. These matrices are famously given for all $n \in \mathbb{N}$ by

$$
S_{n}=\left(\begin{array}{ccccc}
0 & 1 & 0 & \cdots & 0 \\
\vdots & \ddots & \ddots & \ddots & \vdots \\
\vdots & & \ddots & \ddots & 0 \\
0 & \cdots & \cdots & 0 & 1 \\
1 & 0 & \cdots & \cdots & 0
\end{array}\right)
$$

and

$$
C_{n}=\left(\begin{array}{cccc}
1 & & & \\
& z_{n} & & \\
& & \ddots & \\
& & & z_{n}^{n-1}
\end{array}\right),
$$

where $z_{n}=\exp \left(\frac{2 i \pi}{n}\right)$. The smallest $\mathrm{C}^{*}$-algebra containing $C_{n}$ and $S_{n}$ is denoted by $C^{*}\left(S_{n}, C_{n}\right)$, which is the operator norm closure of finite linear combinations of finite products of $C_{n}$ and $S_{n}$ and their conjugate transposes. We note that $S_{n} C_{n}=z_{n} C_{n} S_{n}$, and that both $S_{n}$ and $C_{n}$ are unitary matrices. In fact, the map which sends any function $f: \mathbb{U}_{n} \rightarrow \mathbb{C}$ to the diagonal matrix $f\left(C_{n}\right)$ of entries $f(1), f\left(z_{n}\right), \ldots, f\left(z_{n}^{n-1}\right)$ is a ${ }^{*}$-isomorphism from $C\left(\mathbb{U}_{n}\right)$ to $C^{*}\left(C_{n}\right)$ such that $S_{n} f\left(C_{n}\right) S_{n}^{*}=f \circ r\left(C_{n}\right)$, where $r: z \in$ $\mathbb{U}_{n} \mapsto z_{n} z$. Thus $C^{*}\left(C_{n}, S_{n}\right)$ is also what is known as a $\mathrm{C}^{*}$-crossed product of $\mathbb{U}_{n}$ by the action by translation by itself $\left(\mathrm{C}^{*}\right.$-crossed products are $\mathrm{C}^{*}$-algebraic analogues to semidirect products of groups). When working with finitedimensional models of quantum mechanics, $C_{n}$ and $S_{n}$ are seen as finite-dimensional analogues of the position and momentum observables. In a different direction, they are seen as a matrix model for a certain compactification in string theory. Yet, in a different direction, $t^{\prime}$ Hooft suggests such matrix models as starting points for a new analysis of quantum mechanics. See [Lat16b] for references.

Since $\lim _{n \rightarrow \infty} z_{n}=1$, it is a common heuristics that $C^{*}\left(S_{n}, C_{n}\right)$ is a noncommutative, finite-dimensional approximation for the $\mathrm{C}^{*}$-algebra $C\left(\mathbb{T}^{2}\right)$ of $\mathbb{C}$-valued continuous functions over the 2 -torus $\mathbb{T}^{2}$, seen as the universal $\mathrm{C}^{*}$ algebra generated by two commuting unitaries. Is there indeed a way to make this intuition formal?

An approach to this question is motivated by the observation that it is possible to make rigorous sense of the notion that the groups $\mathbb{U}_{n}$ of $n$th roots of unity in $\mathbb{C}$ converge to a circle as $n$ goes to $\infty$ for the Hausdorff distance induced by the Euclidean distance on the plane (see Figure $1)$. In contrast, the sequence $\left(\left\{\frac{k}{n} \mid k=1, \ldots, n\right\}\right)_{n \in \mathbb{N}^{\prime}}$, whose $n$th entry is homeomorphic to $\mathbb{U}_{n}$ for each $n$, converges to $[0,1]$ for the Hausdorff distance on the real line. Thus, metrics enable us to formally discuss approximations of infinite compact sets by finite sets.

With this motivation in mind, Rieffel proposed to use a suggestion of Connes to define an analogue of a metric for unital $\mathrm{C}^{*}$-algebras that may not be commutative. To this end, we begin with the following question: if $(X, d)$ is a compact metric space, then how do we encode the metric $d$ on the $\mathrm{C}^{*}$-algebra $C(X)$ ? Of course, the metric $d$ induces the Lipschitz seminorm $L_{d}$ on $C(X)$ by setting

$$
L_{d}(f)=\sup _{x, y \in X, x \neq y} \frac{|f(x)-f(y)|}{d(x, y)},
$$

which is 0 only when $f$ is constant. Moreover, $\{f \in C(X) \mid$ $\left.L_{d}(f)<\infty\right\}$ is dense. Furthermore, an observation of L. Kantorovich is that if, for any two Radon probability measures $\mu$ and $\nu$ on $X$, we define $\mathrm{mk}_{L_{d}}(\mu, \nu)$ by

$$
\sup \left\{\left|\int_{X} f d \mu-\int_{X} f d \nu\right| \mid f \in C(X), L_{d}(f) \leq 1\right\},
$$

then $\mathrm{mk}_{L_{d}}$ is a metric which induces the weak* topology on the state space $S(C(X))$ of Radon probability measures over $X$. Moreover, identifying points in $X$ with their associated Dirac point measure gives an isometry from $(X, d)$ into $\left(S(C(X)), \mathrm{mk}_{L_{d}}\right)$. Thus, the Lipschitz seminorm $L_{d}$ encodes the metric $d$ at the level of the $\mathrm{C}^{*}$-algebra $C(X)$, and some of its properties can be made sense of for noncommutative $\mathrm{C}^{*}$-algebras. Rieffel used this as his starting point. Many small variations in the definition of compact quantum metric spaces arose after Rieffel first introduced them in [Rie98]. However, we present the following list of properties which are mostly standard now and are shared by the usual Lipschitz seminorm $L_{d}$.

Definition 2.3 ([Rie98, Rie04, Lat16a, Lat15, Lat16b]). An ordered pair $(A, L)$ of a unital $\mathrm{C}^{*}$-algebra $A$ and a seminorm $L$ defined on a dense subspace $\operatorname{dom}(L)$ of $\left\{a \in A: a=a^{*}\right\}$ is a $(C, D)$-Leibniz compact quantum metric space for some $C \in[1, \infty)$ and $D \in[0, \infty)$ when:

1. $\{a \in \operatorname{dom}(L): L(a)=0\}=\mathbb{R} 1_{A}$,

2. the Monge-Kantorovich metric $\mathrm{mk}_{L^{\prime}}$ defined between any two states $\varphi, \psi \in \mathcal{S}(A)$ by the number $\operatorname{mk}_{L}(\varphi, \psi)$ given by

$$
\sup \{|\varphi(a)-\psi(a)|: a \in \operatorname{dom}(L), L(a) \leq 1\},
$$

induces the weak* topology on the state space $\mathcal{S}(A)$ of A,

3. for all $a, b \in \operatorname{dom}(L)$

$$
\begin{aligned}
\max \left\{L\left(\frac{a b+b a}{2}\right), L\left(\frac{a b-b a}{2 i}\right)\right\} & \\
& \leq C \cdot\left(\|a\|_{A} L(b)+L(a)\|b\|_{A}\right)+D \cdot L(a) L(b),
\end{aligned}
$$

4. $\{a \in \operatorname{dom}(L): L(a) \leq 1\}$ is closed in $A$. 
In 3, the usual Leibniz rule is given by $C=1$ and $D=0$, but as we shall see later (Theorem 3.5), examples of compact quantum metric spaces with different values occur, and without allowing for these different values, we will not be able to produce many convergence results.

The noncommutative $\mathrm{C}^{*}$-algebra $C^{*}\left(S_{n}, C_{n}\right)$ can be endowed with a quantum metric structure. To this end, let us fix any continuous length function $\ell$ on $\mathbb{T}^{2}$. For instance, we can choose $\ell$ to be defined by setting $\ell(\omega)$ for all $\omega=\left(z, z^{\prime}\right) \in \mathbb{T}^{2}$ as

$$
\min \left\{\sqrt{x^{2}+y^{2}}: z=\exp (2 i \pi x), z^{\prime}=\exp (2 i \pi y)\right\} .
$$

The function $\left(\omega, \omega^{\prime}\right) \in \mathbb{T}^{2} \times \mathbb{T}^{2} \mapsto \ell\left(\omega^{-1} \omega^{\prime}\right)$ is thus a metric $d_{\ell}$ on the 2-torus $\mathbb{T}^{2}$, which induces a Lipschitz seminorm $L_{d_{\ell}}$ on $C\left(\mathbb{T}^{2}\right)$. On the other hand, Rieffel defined in [Rie98], for any $a \in C^{*}\left(S_{n}, C_{n}\right)$ :

$$
L_{n}(a)=\sup \left\{\frac{\left\|\alpha^{w}(a)-a\right\|_{C^{*}\left(S_{n}, C_{n}\right)}}{\ell(w)} \mid w \in \mathbb{U}_{n}^{2}\right\},
$$

where $\alpha$ is the so-called dual action of $\mathbb{U}_{n}^{2}$ on $C^{*}\left(S_{n}, C_{n}\right)$ uniquely defined by $\alpha^{w_{1}, w_{2}}\left(C_{n}\right)=w_{1} C_{n}$ and $\alpha^{w_{1}, w_{2}}\left(S_{n}\right)=$ $w_{2} S_{n}$. Then $\left(C^{*}\left(S_{n}, C_{n}\right), L_{n}\right)$ is a compact quantum metric space.

To discuss the convergence of $\left(C^{*}\left(S_{n}, C_{n}\right), L_{n}\right)_{n \in \mathbb{N}}$ to $\left(C\left(\mathbb{T}^{2}\right), L_{d_{\ell}}\right)$, we generalize the intrinsic version of the Hausdorff distance introduced by M. Gromov [Gro81] (see the following definition), since, of course, noncommutative algebras are not quotients of commutative ones.

Definition 2.4. The Gromov-Hausdorff distance between any two compact metric spaces $(X, d)$ and $\left(Y, d^{\prime}\right)$ is

$$
\begin{gathered}
\mathrm{GH}\left((X, d),\left(Y, d^{\prime}\right)\right)=\inf \left\{\text { Haus }_{d^{\prime \prime}}(j(X), g(Y)) \mid\right. \\
\left(Z, d^{\prime \prime}\right) \text { is a compact metric space and }
\end{gathered}
$$$$
j: X \hookrightarrow Z, g: Y \hookrightarrow Z \text { are isometries\}. }
$$

We note that if $X, Y \subseteq Z$, where $(Z, d)$ is a compact metric space, then

$$
\mathrm{GH}((X, d),(Y, d)) \leq \operatorname{Haus}_{d}(X, Y) .
$$

Now, the Gromov-Hausdorff distance is a distance on the class of all compact metric spaces, up to isometrymeaning that $\operatorname{GH}\left((X, d),\left(Y, d^{\prime}\right)\right)=0$ if and only if $(X, d)$ and $\left(Y, d^{\prime}\right)$ are isometric. This distance has also found applications in Riemannian geometry [Gro81].

Rieffel used Gromov's idea to define a first noncommutative analogue of the Gromov-Hausdorff distance between compact quantum metric spaces. Rieffel's quantum Gromov-Hausdorff distance is a complete pseudometric, which is zero exactly between quantum compact metric spaces whose state spaces are isometric, with an isometry implemented by the dual of a linear map. However, this does not imply that the underlying $\mathrm{C}^{*}$-algebras are ${ }^{*}$-isomorphic. Variations of Rieffel's construction were offered (by D. Kerr and H. Li-see [KL09] and the references therein-and the third author, to name a few) to address this coincidence property issue. A variation due to the third author solved this problem by accounting for the Leibniz property, used as a means to connect the algebraic structure and the quantum metric structure, and a property which gained importance as research in noncommutative metric geometry progressed.

Rieffel indeed observed in [Rie04, Proposition 3.1], as an essential component of his construction, that if $\pi$ : $A \rightarrow B$ is a unit-preserving surjective ${ }^{*}$-morphism, where $(A, L)$ and $\left(B, L^{\prime}\right)$ are compact quantum metric spaces, then the dual map $\pi^{*}: \varphi \in S(B) \mapsto \varphi \circ \pi \in S(A)$ induced by $\pi$ between the state spaces of $B$ and $A$ is an isometry from $\left(S(B), \mathrm{mk}_{L^{\prime}}\right)$ to $\left(S(A), \mathrm{mk}_{L}\right)$ if and only if $L^{\prime}(b)=\inf \{L(a)$ : $\pi(a)=b\}$ for all $b$ in the domain of $L^{\prime}$. We are led to the following definition.

Definition 2.5 ([Lat15, Lat16b]). A quantum isometry $\pi$ : $(A, L) \rightarrow\left(B, L^{\prime}\right)$ is a surjective unital ${ }^{*}$-morphism such that

$$
\forall b \in \operatorname{dom}\left(L^{\prime}\right) \quad L^{\prime}(b)=\inf \{L(a): \pi(a)=b\} .
$$

A full quantum isometry is a ${ }^{*}$-isomorphism (bijective *morphism) such that $L^{\prime} \circ \pi=L$.

Following Gromov's and Rieffel's prescription with the Leibniz property in mind and the above definition of a quantum isometry, the third author introduced in [Lat16a, Lat15] a new analogue of the Gromov-Hausdorff distance called the propinquity, described as follows. Fix $(C, D) \in[1, \infty) \times[0, \infty)$. A tunnel $\tau=\left(B, L, \pi_{1}, \pi_{2}\right)$ between two $(C, D)$-Leibniz compact quantum metric spaces $\left(A_{1}, L_{1}\right)$ and $\left(A_{2}, L_{2}\right)$ is given by a $(C, D)$-Leibniz compact quantum metric space $(B, L)$ and two quantum isometries $\pi_{1}:(B, L) \rightarrow\left(A_{1}, L_{1}\right)$ and $\pi_{2}:(B, L) \rightarrow\left(A_{2}, L_{2}\right)$. The extent $\chi(\tau)$ of the tunnel $\tau$ is then given by

$$
\max _{j \in\{1,2\}} \operatorname{Haus}_{\mathrm{mk}_{L}}\left(S(B), \pi_{j}^{*}\left(S\left(A_{j}\right)\right)\right) .
$$

The third author then introduced the GromovHausdorff propinquity as follows.

Definition 2.6 ([Lat15, Lat16b]). Fix $(C, D) \in[1, \infty) \times$ $[0, \infty)$. The Gromov-Hausdorff propinquity between two $(C, D)$-Leibniz compact quantum metric spaces $\left(A_{1}, L_{1}\right)$ and $\left(A_{2}, L_{2}\right)$ is defined by

$$
\begin{aligned}
& \Lambda_{(C, D)}^{*}\left(\left(A_{1}, L_{1}\right),\left(A_{2}, L_{2}\right)\right)=\inf \{\chi(\tau) \mid \\
& \left.\tau \text { is a tunnel between }\left(A_{1}, L_{1}\right) \text { and }\left(A_{2}, L_{2}\right)\right\} .
\end{aligned}
$$

The third author proved that $\Lambda_{(C, D)}^{*}$ is a complete metric up to full quantum isometry on the class of $(C, D)$-Leibniz compact quantum metric spaces. Thus, $\Lambda_{(C, D)}^{*}$ preserves both the algebraic and quantum metric structure. Moreover, when we identify a compact metric space $(X, d)$ with the compact quantum metric space $\left(C(X), L_{d}\right)$, then the restriction of $\Lambda_{(C, D)}^{*}$ to the class of compact metric spaces 
induces the same topology as the Gromov-Hausdorff distance $\mathrm{GH}$. In particular, we have that

$$
\Lambda_{(C, D)}^{*}\left(\left(C(X), L_{d}\right),\left(C(Y), L_{d^{\prime}}\right)\right) \leq \mathrm{GH}\left((X, d),\left(Y, d^{\prime}\right)\right) \text {. }
$$

The third author then proved that $\left(C^{*}\left(S_{n}, C_{n}\right), L_{n}\right)$ converges to $\left(C\left(\mathbb{T}^{2}\right), L_{d_{\ell}}\right)$ for the Gromov-Hausdorff propinquity. Thus, we were able to formalize that the clock and shift matrices generate a finite-dimensional approximation of the $\mathrm{C}^{*}$-algebra $C\left(\mathbb{T}^{2}\right)$, as we wished.

There are many other examples of compact quantum metric spaces and convergence for the propinquity. For instance, we could work with $C^{*}\left(S_{n}, C_{n}\right)$ where $z_{n}$ is just any $n$-root of unity, and prove that with a similar construction $C^{*}\left(S_{n}, C_{n}\right)$ converges to a noncommutative/quantum 2-torus when $\left(z_{n}\right)_{n \in \mathbb{N}_{0}}$ converges to some $z=\exp (2 \pi i \theta) \in$ $\mathbb{T}$, where the quantum 2-torus here would be the universal $\mathrm{C}^{*}$-algebra generated by two unitaries $U$ and $V$ such that

$$
U V=z V U,
$$

denoted by $\mathcal{A}_{\theta}$ and also called a rotation algebra, where for $\theta=0, \mathcal{A}_{0} \cong C\left(\mathbb{T}^{2}\right)$. (See [Lat16a, Lat15], where this was also generalized further, to prove the continuity of the family of quantum 2-tori in Gromov-Hausdorff propinquity, or to allow for more than two unitary generators. For another direction, see [JRZ18], where the authors use a harmonic analysis approach to convergence of the quantum tori with respect to the Gromov-Hausdorff propinquity.) Other examples include approximating spheres with fuzzy spheres (which are finite-dimensional $\mathrm{C}^{*}$-algebras) using the propinquity, as done by Rieffel, which is also a common example from the physics literature [Rie16].

Among interesting examples, the first and third authors studied another class of $\mathrm{C}^{*}$-algebras, from this metric perspective: the approximately finite-dimensional algebras, or AF-algebras [AL15]. AF-algebras, introduced by Bratteli [Dav96, Chapter III], actually arose from considerations from quantum statistical mechanics. They also were the first class of $\mathrm{C}^{*}$-algebras which was fully classified, up to ${ }^{*}$-isomorphism, by an invariant called the Elliott invariant, which involved the $K$-theory of the $\mathrm{C}^{*}$-algebra. This spawned the Elliott classification program (see [EE93] for one of the most important classes of $\mathrm{C}^{*}$-algebras classified by this invariant, and which contains the irrational rotation algebras of equation (4)).

AF-algebras come as limits, in the categorical sense, of finite-dimensional $\mathrm{C}^{*}$-algebras. Are they, as well, limits of their inductive sequences for the Gromov-Hausdorff propinquity? This question includes the problem of finding appropriate quantum metrics on AF-algebras. Once this is done, many other intriguing topological questions can be asked about the class of AF-algebras. In fact, the main convergence result we present about AF-algebras is motivated by the convergence of the rotation algebras described in the previous paragraph since certain rotation algebras can be embedded into AF-algebras. An approach to this convergence of AF-algebras was given by the first author in [Agu19] using a type of convergence of ideals of AFalgebras. This is what is being represented in the opening graphic, where the ideals $I_{\theta_{n}}$ (represented in green) are approaching the ideal $I_{\theta}$ in this topology on ideals. We will provide a more detailed description of this graphic later. Moreover, AF-algebras produce instructive examples in the theory of quantum metric spaces using the Cantor set in [AL19] and matrices in [AB19].

\section{The Metric Geometry of the Class of Approximately Finite-Dimensional Algebras}

We already saw examples of finite-dimensional $\mathrm{C}^{*}$ algebras in the form of $M_{n}(\mathbb{C})$ and $C^{*}\left(S_{n}, C_{n}\right)$ (a unital $\mathrm{C}^{*}$-subalgebra of $M_{n}(\mathbb{C})$ ), but now, we state that, in general, all finite-dimensional $\mathrm{C}^{*}$-algebras are given by the structure of $M_{n}(\mathbb{C})$. Indeed, If $A$ is finite dimensional, then there exist $N \in \mathbb{N}, n_{1}, \ldots, n_{N} \in \mathbb{N}$, such that $A \cong$ $\bigoplus_{k=1}^{N} M_{n_{k}}(\mathbb{C})$, where the ${ }^{*}$-algebra is given by coordinatewise operations and the norm is the max norm over the operator matrix norm in each coordinate.

Now, approximately finite-dimensional $\mathrm{C}^{*}$-algebras (AF-algebras) are inductive limits of finite-dimensional $\mathrm{C}^{*}$ algebras. Inductive limits always exist in the category of $\mathrm{C}^{*}$-algebras, and, up to ${ }^{*}$-isomorphism, can be described in the following simple terms.

Definition 3.1. A C*-algebra $A$ is an inductive limit of $a$ sequence of $C^{*}$-algebras $\left(A_{n}\right)_{n \in \mathbb{N}_{0}}$ if for all $n \in \mathbb{N}_{0}, A_{n}$ is a $\mathrm{C}^{*}$-subalgebra of $A, A_{n} \subseteq A_{n+1}$, and $\overline{\bigcup_{n \in \mathbb{N}_{0}} A_{n}}\|\cdot\|_{A}=A$.

We call $A$ an approximately finite-dimensional $C^{*}$-algebra (AF-algebra) if it is the inductive limit of a sequence of $\mathrm{C}^{*}$ algebras satisfying $\operatorname{dim}\left(A_{n}\right)<\infty$ for all $n \in \mathbb{N}_{0}$.

Not all $\mathrm{C}^{*}$-algebras are AF-algebras: for instance, the rotation algebras $\mathcal{A}_{\theta}$ from (4), including $C\left(\mathbb{T}^{2}\right)$, are not AF. Of course, any finite-dimensional $\mathrm{C}^{*}$-algebra $A$ is $\mathrm{AF}$, by simply choosing the constant sequence $(A)_{n \in \mathbb{N}_{0}}$ in Definition 3.1 .

In Example 3.2, a well-known example of an AFalgebra is given by the $\mathrm{C}^{*}$-algebra of $\mathbb{C}$-valued continuous functions over the Cantor space $\mathcal{C}$, where $\mathcal{C}=$ $\prod_{n \in \mathbb{N}_{0}}\{0,1\}$ equipped with the product topology (each $\{0,1\}$ is equipped with the discrete topology); this space is homeomorphic to the middle-thirds Cantor set.

Example 3.2. For each $n \in \mathbb{N}_{0}$, we define $\eta_{n} \in C(\mathcal{C})$ by $\eta_{n}:\left(z_{m}\right)_{m \in \mathbb{N}_{0}} \in \mathcal{C} \mapsto z_{n}$. Also, define $u_{n} \in C(\mathcal{C})$ by $u_{n}=2 \eta_{n}-1_{C(\mathcal{C})}$. Set $A_{0}=\mathbb{C} 1_{C(\mathcal{C})}$. For each $n \in \mathbb{N}$, set $A_{n}=C^{*}\left(u_{0}, \ldots, u_{n-1}\right)$. The $\mathrm{C}^{*}$-algebra $C(\mathcal{C})$ is an AFalgebra since $\operatorname{dim}\left(A_{n}\right)=2^{n}$ for all $n \in \mathbb{N}_{0}$ and $C(\mathcal{C})=$ $\bar{\bigcup}_{n \in \mathbb{N}_{0}} A_{n}\left\|^{\|} \cdot\right\|_{C(e)}$. 


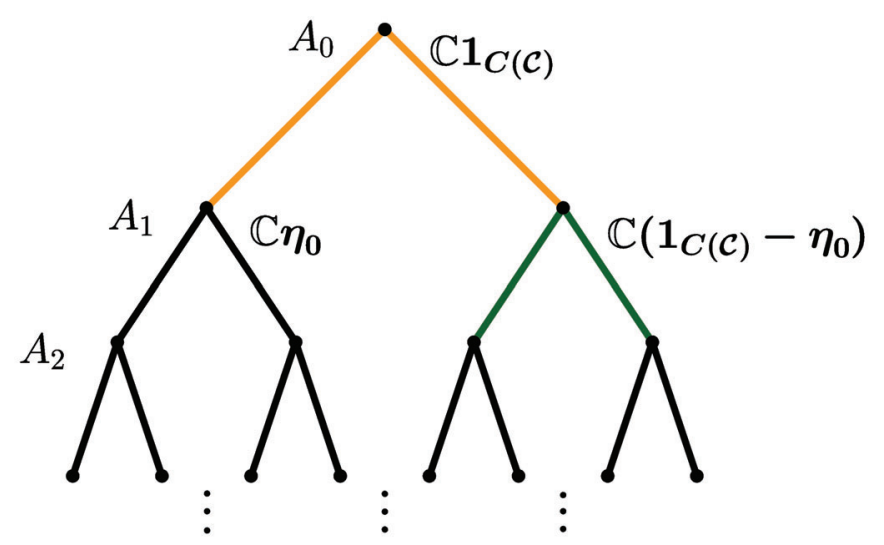

Figure 2. A Bratteli diagram for $C(\mathcal{C})$.

Another standard picture of the Cantor space is as the space of infinite paths in the infinite binary tree. Bratteli associated certain infinite graphs to each AF-algebra called Bratteli diagrams. Although there is no unique Bratteli diagram associated to a given AF-algebra, the relation of a *-isomorphism between AF-algebras translates to a natural equivalence relation between Bratteli diagrams.

As an example, a Bratteli diagram for $C(\mathcal{C})$ is, indeed, given by the infinite binary tree built by the sequence $\left(A_{n}\right)_{n \in \mathbb{N}_{0}}$ from Example 3.2 (see Figure 2). The way a Bratteli diagram is built is as follows: each horizontal row of vertices represents an $A_{n}$, and the rows are ordered from top to bottom. Recall that since $\operatorname{dim}\left(A_{n}\right)<\infty, A_{n}$ can be written as a direct sum of $M_{k}(\mathbb{C})$ 's; each vertex represents a term in this direct sum. The edges represent an embedding from $A_{n}$ into $A_{n+1}$ (we do not state a description of how the edges are constructed, but we will show how this works in our examples). To see how to construct the Bratteli diagram in Figure 2, we first note that $A_{1}$ and $A_{2}$ can be written as $\mathbb{C} \eta_{0} \oplus \mathbb{C}\left(1_{C(\mathcal{C})}-\eta_{0}\right)$ and $\mathbb{C} \eta_{0} \eta_{1} \oplus$ $\mathbb{C} \eta_{0}\left(1_{C(\mathcal{C})}-\eta_{1}\right) \oplus \mathbb{C}\left(1_{C(\mathcal{C})}-\eta_{0}\right) \eta_{1} \oplus \mathbb{C}\left(1_{C(\mathcal{C})}-\eta_{0}\right)\left(1_{C(\mathcal{C})}-\right.$ $\left.\eta_{1}\right)$, respectively. Each term in the direct summand is $*_{-}$ isomorphic to $\mathbb{C}=M_{1}(\mathbb{C})$, and recall that $A_{0}=\mathbb{C} 1_{C(\mathcal{C})}$. Now, $A_{0}, A_{1}$, and $A_{2}$ determine the vertices from top to bottom. The edges are given by the relationships between the generators. For example, since $1_{C(\mathcal{C})}=\eta_{0}+\left(1_{C(\mathcal{C})}-\eta_{0}\right)$, we have an embedding from $A_{0}$ into $A_{1}$ given by $r 1_{C(\mathcal{C})} \in$ $A_{0} \mapsto\left(r \eta_{0}, r\left(1_{C(\mathcal{C})}-\eta_{0}\right)\right) \in A_{1}$, which is represented by the gold edges. Similarly, the green edges are given by $\left(1_{C(\mathcal{C})}-\eta_{0}\right)=\left(1_{C(\mathcal{C})}-\eta_{0}\right) \eta_{1}+\left(1_{C(\mathcal{C})}-\eta_{0}\right)\left(1_{C(\mathcal{C})}-\eta_{1}\right)$, and so on.

Bratteli also showed that one can construct AF-algebras from Bratteli diagrams, which has given rise to many new and fascinating $\mathrm{C}^{*}$-algebras. For instance, Effros and Shen introduced AF-algebras constructed from continued fraction expansions of irrationals, which were then used by Pimnser and Voiculescu to classify the rotation algebras $\mathcal{A}_{\theta}$ for irrational $\theta$ (see equation (4) and [Dav96, Chapter VI] and references therein). This classification can be captured by the classification of the irrational rotational algebras by the Elliott invariant in [EE93], where continued fractions also played a pivotal role. Now, the Effros-Shen algebras were some of the first examples of AF-algebras equipped with a quantum metric in [AL15]. Another interesting example, related to the rotation algebras, is the Boca-Mundici algebra, $\mathfrak{F}$, which is built using properties of the Farey tessellation and continued fraction expansions of irrationals [Boc08]. In particular, for each irrational $\theta \in(0,1)$, there exists an ideal $I_{\theta} \subseteq \mathfrak{F}$ and an injective * homomorphism $\pi_{\theta}: \mathcal{A}_{\theta} \rightarrow \mathfrak{F} / I_{\theta}$, where $\mathcal{A}_{\theta}$ is the rotation algebra associated to $\theta$. (The $\mathfrak{F} / I_{\theta}$ 's are also ${ }^{*}$-isomorphic to the Effros-Shen algebras.) This is relevant to the work in [Agu19] and the opening graphic. We will discuss this and ideals of $\mathrm{C}^{*}$-algebras more later, but now, we present the construction of $\mathfrak{F}$ from a Bratteli diagram.

Example 3.3. The Boca-Mundici algebra $\mathfrak{F}$ is constructed using a Bratteli diagram given by the pattern established in Figure 3. The top vertex represents $\mathfrak{F}_{0} \cong \mathbb{C}$. The next level below is $\mathfrak{F}_{1} \cong \mathbb{C} \oplus \mathbb{C}$. The edges between $\mathfrak{F}_{0}$ and $\mathfrak{F}_{1}$ are determined by the embedding $r \in \mathbb{C} \mapsto(r, r) \in \mathbb{C} \oplus \mathbb{C}$. Next, we have $\mathfrak{F}_{2} \cong \mathbb{C} \oplus M_{2}(\mathbb{C}) \oplus \mathbb{C}$, and the edges between $\mathfrak{F}_{1}$ and $\mathfrak{\mho}_{2}$ are given by the embedding

$$
(r, t) \in \mathbb{C} \oplus \mathbb{C} \mapsto\left(r,\left[\begin{array}{ll}
r & 0 \\
0 & t
\end{array}\right], t\right) \in \mathbb{C} \oplus M_{2}(\mathbb{C}) \oplus \mathbb{C},
$$

and the path that the $r$ takes is given by the gold edges. The green edges show how the $M_{2}(\mathbb{C})$ in $\mathfrak{\mho}_{2}$ embeds into the $M_{3}(\mathbb{C}) \oplus M_{2}(\mathbb{C}) \oplus M_{3}(\mathbb{C})$ of $\mathfrak{F}_{3} \cong \mathbb{C} \oplus M_{3}(\mathbb{C}) \oplus M_{2}(\mathbb{C}) \oplus$ $M_{3}(\mathbb{C}) \oplus \mathbb{C}$. The rest of the construction of $\mathfrak{F}$ follows this pattern for all $n \in \mathbb{N}$.

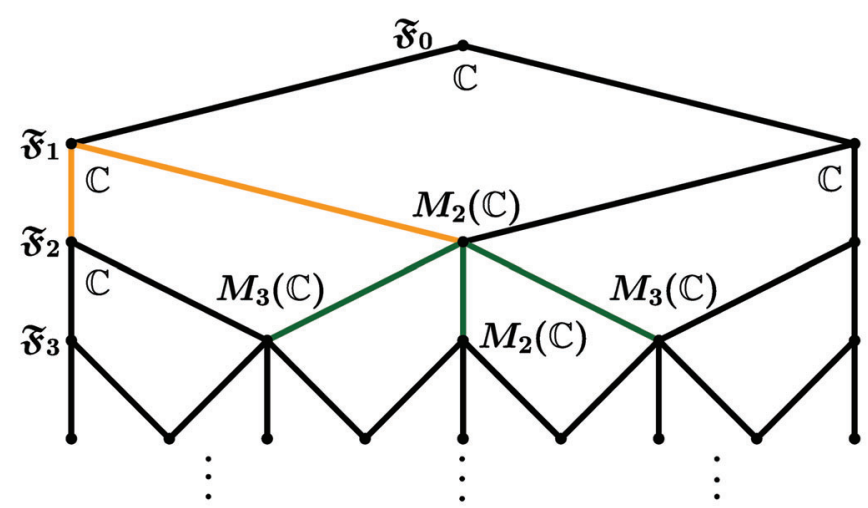

Figure 3. A Bratteli diagram for $\mathfrak{F}$.

It is natural to ask whether AF-algebras can be endowed with a quantum metric, and discuss whether, in particular, they are limits of their defining inductive sequences, in the sense of the propinquity. This will not only provide us with interesting examples of convergence in GromovHausdorff propinquity, but also provide two informative examples in the theory of quantum metric spaces. 
We will present the construction of these quantum metrics in the case when a unital AF-algebra $A$ has a faithful tracial state (a state $\mu$ that satisfies $\mu\left(a^{*} a\right)=0 \Longrightarrow a=0$ (faithful) and $\mu(a b)=\mu(b a)$ (tracial) for all $a, b \in A$ ). For $M_{n}(\mathbb{C})$, the normalized trace $\frac{1}{n} \cdot \operatorname{Tr}$ is a faithful tracial state. This covers many unital AF-algebras including the examples presented above and all simple AF-algebras. This construction has been generalized for all unital AF-algebras by the first author, but we do not present this here. The advantage of a faithful tracial state is that it allows us to build conditional expectations.

Definition 3.4. Let $A$ be a unital $C^{*}$-algebra, and let $B \subseteq A$ be a unital $C^{*}$-subalgebra of $A$. A contractive linear map $E: A \rightarrow B$ is a conditional expectation of $A$ onto $B$ if $E(b)=b$ for all $b \in B$.

We can use conditional expectations to quantify how far an element of an AF-algebra is from one of its finitedimensional subalgebras, which is the basis for the following quantum metric on AF-algebras.

Theorem 3.5 ([AL15]). Let $A$ be a unital AF-algebra given by a sequence of unital finite-dimensional $C^{*}$-subalgebras $U=$ $\left(A_{n}\right)_{n \in \mathbb{N}_{0}}$ such that $A_{0}=\mathbb{C 1}_{A}$. Assume that $A$ has a faithful tracial state $\tau$. For each $n \in \mathbb{N}_{0}$, let $E_{n}: A \rightarrow A_{n}$ be the unique conditional expectation satisfying $\tau\left(E_{n}(a)\right)=\tau(a)$ for all $a \in A$. Let $(\beta(n))_{n \in \mathbb{N}_{0}}$ be a sequence of positive real numbers converging to 0 .

If for each $a \in A$, we set

$$
L_{U, \tau}^{\beta}(a)=\sup _{n \in \mathbb{N}_{0}} \frac{\left\|a-E_{n}(a)\right\|_{A}}{\beta(n)},
$$

then $\left(A, L_{U, \tau}^{\beta}\right)$ and $\left(A_{n}, L_{U, \tau}^{\beta}\right)$ for all $n \in \mathbb{N}_{0}$ are (2,0)-Leibniz compact quantum metric spaces such that $\Lambda_{(2,0)}^{*}\left(\left(A_{n}, L_{U, \tau}^{\beta}\right),\left(A, L_{U, \tau}^{\beta}\right)\right) \leq \beta(n)$ for all $n \in \mathbb{N}_{0}$, and thus

$$
\lim _{n \rightarrow \infty} \Lambda_{(2,0)}^{*}\left(\left(A_{n}, L_{U, \tau}^{\beta}\right),\left(A, L_{U, \tau}^{\beta}\right)\right)=0 .
$$

For our first example, we consider the Cantor space $\mathcal{C}$ and the standard metric $d_{\mathcal{C}}$ on $\mathcal{C}$ defined for all $x, y \in \mathcal{C}$ by

$$
d_{\mathcal{C}}(x, y)= \begin{cases}0, & x=y, \\ 2^{-\min \left\{m \mid x_{m} \neq y_{m}\right\}}, & \text { otherwise, }\end{cases}
$$

which induces the Lipschitz seminorm $L_{d_{\mathcal{e}}}$ on $C(\mathcal{C})$. On the other hand, if we let $\lambda$ be the faithful tracial state on $C(\mathcal{C})$ given by integration with the unique Haar measure on the compact group $\mathcal{C}$, let $\mathcal{T}_{\mathcal{C}}$ denote the inductive sequence given in Example 3.2, and let $\left(\beta_{\mathcal{C}}(n)\right)_{n \in \mathbb{N}_{0}}=$ $\left(2^{-(n+1)}\right)_{n \in \mathbb{N}_{0}}$, we have another Lip-norm $L_{\mathcal{T}_{\mathcal{e}}, \lambda}^{\beta_{e}}$ on $C(\mathcal{C})$ given by Theorem 3.5. First, we note the following relationship between these two Lip-norms. Denote the Dirac point masses defined for each $x \in \mathcal{C}$ by $\delta_{x}: f \in C(\mathcal{C}) \mapsto$ $f(x) \in \mathbb{C}$.
Theorem 3.6 ([AL15]). For each $x, y \in \mathcal{C}$, we have

$$
\mathrm{mk}_{L_{\mathcal{J}_{e}, \lambda}^{\beta_{\mathcal{C}}}}\left(\delta_{x}, \delta_{y}\right)=d_{\mathcal{C}}(x, y)=\mathrm{mk}_{L_{d_{e}}}\left(\delta_{x}, \delta_{y}\right)
$$

However, while this shows that the Lip-norm $L_{\mathcal{T}_{e}, \lambda}^{\beta_{e}}$ induces this standard ultrametric on the Cantor space, $L_{\mathcal{T}_{e}, \lambda}^{\beta_{e}}$ is not the Lipschitz seminorm induced by this metric, $L_{d_{e}}$. This is given by the following theorem, where we also observe another striking relationship between $L_{\mathcal{T}_{\mathcal{e}}, \lambda}^{\beta_{\mathcal{C}}}$ and $L_{d_{\mathcal{e}}}$. Indeed:

Theorem 3.7 ([AL19]). For each $j \in \mathbb{N}_{0}$, it holds that $L_{d_{\mathcal{e}}}\left(u_{j}\right)=2^{j+1}=L_{\mathcal{T}_{\mathcal{e}}, \lambda}^{\beta_{e}}\left(u_{j}\right)$. And, for each $z \in \mathbb{N}, j_{0}, \ldots, j_{z} \in$ $\mathbb{N}_{0}$ such that $j_{0}<\cdots<j_{z}$ it holds that

$$
L_{d_{\mathcal{C}}}\left(u_{j_{0}} \cdots u_{j_{z}}\right)=2^{j_{z}+1}=L_{\mathcal{J}_{\mathcal{e}}, \lambda}^{\beta_{\mathcal{C}}}\left(u_{j_{0}} \cdots u_{j_{z}}\right) .
$$

Therefore $L_{d_{\mathcal{C}}}$ and $L_{\mathcal{T}_{\mathcal{e}}, \lambda}^{\beta_{\mathcal{C}}}$ agree on a Hamel basis for the dense subspace $\bigcup_{n \in \mathbb{N}_{0}} A_{n}$.

However, if $\alpha_{0}, \alpha_{1}, \alpha_{2} \in \mathbb{R}$ such that $\alpha_{0}>\alpha_{1}+\alpha_{2}$ and $\alpha_{1}=\alpha_{2}>0$, and we set $f=\alpha_{0} u_{0}+\alpha_{2} u_{1}+\alpha_{2} u_{0} u_{1}$, then $L_{d_{\mathcal{C}}}(f)=\max \left\{2\left(\alpha_{0}+\alpha_{1}\right), 8 \alpha_{1}\right\}, L_{\mathcal{T}_{\mathcal{e}}, \lambda}^{\beta_{\mathcal{C}}}(f)=2\left(\alpha_{0}+2 \alpha_{1}\right)$, and

$$
L_{d_{\mathcal{e}}}(f)<L_{\mathcal{T}_{\mathcal{e}}, \lambda}^{\beta_{\mathcal{C}}}(f) .
$$

The Lip-norms $L_{d_{\mathcal{C}}}$ and $L_{\mathcal{T}_{\mathcal{e}}, \lambda}^{\beta_{\mathcal{C}}}$ are different, which reflects the fact that Lip-norms define metrics on the entire state space of a $\mathrm{C}^{*}$-algebra, not just on the extreme points of the state space (pure states) (which correspond, in the commutative case, to the actual points in the spectrum of the $\mathrm{C}^{*}$-algebra by the Dirac point masses); therefore, in general, there are Lip-norms which are defined on $C(X)$ for $X$ compact metrizable which do not arise as Lipschitz seminorms for any metric on $X$. The reason for this is that, for noncommutative $\mathrm{C}^{*}$-algebras, there is no natural notion of points, and, in fact, the set of extreme points of the state space equipped with the weak* topology is typically not compact, and can be quite complicated (even dense in the state space).

For our next example, the quantum metrics induced on finite-dimensional $\mathrm{C}^{*}$-algebras from Theorem 3.5 provide nontrivial examples of nonisometric quantum metric spaces for which the underlying $\mathrm{C}^{*}$-algebras are ${ }^{*}$ isomorphic. Indeed, while computing lower bounds for the propinquity is typically difficult, we have the following result, which also shows that the Lip-norms of Theorem 3.5 are sensitive to the particular inductive sequence that is chosen.

Theorem 3.8 ([AB19]). Fix $n \in \mathbb{N}$ such that $n$ is not prime. Let $k \in \mathbb{N} \backslash\{1\}$ such that $k$ divides $n$. Let $A_{k}=\left\{\operatorname{blockdiag}\left(a_{1}, \ldots, a_{n / k}\right) \mid \forall m, p \in\{1, \ldots, n / k\}, a_{m} \in\right.$ $\left.M_{k}(\mathbb{C}), a_{m}=a_{p}\right\}$, which is a unital $C^{*}$-subalgebra of $M_{n}(\mathbb{C})$ also denoted by $I_{n} \otimes M_{k}(\mathbb{C})$ in Kronecker product notation. Consider the inductive sequences $U_{1}=\left(\mathbb{C} I_{n}, M_{n}(\mathbb{C}), M_{n}(\mathbb{C}), \ldots\right)$, 
$U_{2}=\left(\mathbb{C I}_{n}, A_{k}, M_{n}(\mathbb{C}), M_{n}(\mathbb{C}), \ldots\right)$, and the faithful tracial state $\tau_{n}=\frac{1}{n} \cdot$ Tr. Set $(\beta(n))_{n \in \mathbb{N}_{0}}=\left(1 / k^{n}\right)_{n \in \mathbb{N}_{0}}$. It holds that

$$
\Lambda_{(2,0)}^{*}\left(\left(M_{n}(\mathbb{C}), L_{U_{1}, \tau_{n}}^{\beta}\right),\left(M_{n}(\mathbb{C}), L_{U_{2}, \tau_{n}}^{\beta}\right)\right)>0 .
$$

Next, we move on to convergence of AF-algebras in propinquity. In [AL15], the family of Effros-Shen algebras were shown to be continuous for the propinquity with respect to their irrational parameters, once endowed with the quantum metrics from Theorem 3.5. Now, recall that these $\mathrm{C}^{*}$-algebras are all quotients of the Boca-Mundici algebra, $\mathfrak{F}$. Furthermore, there exist interesting topologies on the space of ideals of a $\mathrm{C}^{*}$-algebra, and, in particular, the Fell topology of J. M. G. Fell in [Fel61]. Therefore, the following question arises: if we endow each $\mathfrak{F} / I_{\theta}$ with a quantum metric from Theorem 3.5, then is the natural map from the ideal space of the Boca-Mundici algebra to the space of Effros-Shen algebras, seen as quotients of the Boca-Mundici algebra, continuous from the Fell topology to the propinquity topology? Moreover, as each $\mathfrak{F} / I_{\theta}$ is built by an irrational number, the question of continuity of the quotients with respect to their irrational parameters is compulsory. What is surprising is that continuity of these quotients with respect to ideals or irrationals will be given simultaneously due to the Fell topology. But, first:

Theorem 3.9 (Segal). Let $A$ be a $C^{*}$-algebra. If $I \subseteq A$ is a norm-closed two-sided ideal of $A$, then $I$ is self-adjoint and thus $a C^{*}$-subalgebra of $A$. Furthermore, the quotient space $A / I$ is a $C^{*}$-algebra with quotient norm and adjoint $(a+I)^{*}=a^{*}+I$.

It is quite surprising and useful that the norm-closed two-sided ideals of a $\mathrm{C}^{*}$-algebra are automatically $\mathrm{C}^{*}$ subalgebras and that, furthermore, we get $\mathrm{C}^{*}$-algebras from the quotient spaces, which leads us to the following definition.

Definition 3.10. Let $A$ be a $C^{*}$-algebra. The ideal space of $A$, Ideals $(A)$, is the set of norm-closed two-sided ideals of $A$ including $\{0\}$ and $A$.

Example 3.11. Let $X$ be a compact Hausdorff space. For each closed $F \subseteq X$, we have that

$$
I_{F}=\{f \in C(X) \mid \forall x \in F, f(x)=0\} \in \operatorname{Ideals}(C(X))
$$

and $C(X) / I_{F} \cong C(F)$. Also, $I \in \operatorname{Ideals}(C(X))$ if and only if there exists a closed $F \subseteq X$ such that $I=I_{F}$.

Next, ideals and quotients of a fixed AF-algebra $A$ can be determined from any Bratteli diagram given for $A$ [Dav96, Section III.4]. The opening graphic and Figure 4 display this in the ideals $I_{\theta}$ of $\mathfrak{F}$ associated to irrational numbers. The ideal is presented by combining both shades of green and the quotient is presented combining both shades of gold (we will discuss the shading in a moment). Another important aspect of the Bratteli diagram for $\mathfrak{F}$ is that, after the top vertex each horizontal level of vertices determines a partition of $[0,1]$ with the vertices as rational endpoints and the leftmost vertex is always 0 , the middle vertex is always $1 / 2$ (starting at $\mathfrak{F}_{2}$ ), and the rightmost vertex is always 1 (the remaining spacing of vertices is not drawn to scale in Figure 3 or Figure 4 ), and the ideal $I_{\theta}$ determines where $\theta$ lies in each partition. Thus, the green is consuming the diagram as $n \rightarrow \infty$ since it is determining the smaller and smaller intervals for which $\theta$ lies, and, due to this, each $I_{\theta}$ determines an irrational number uniquely, which is related to the unique continued fraction expansion of $\theta$.
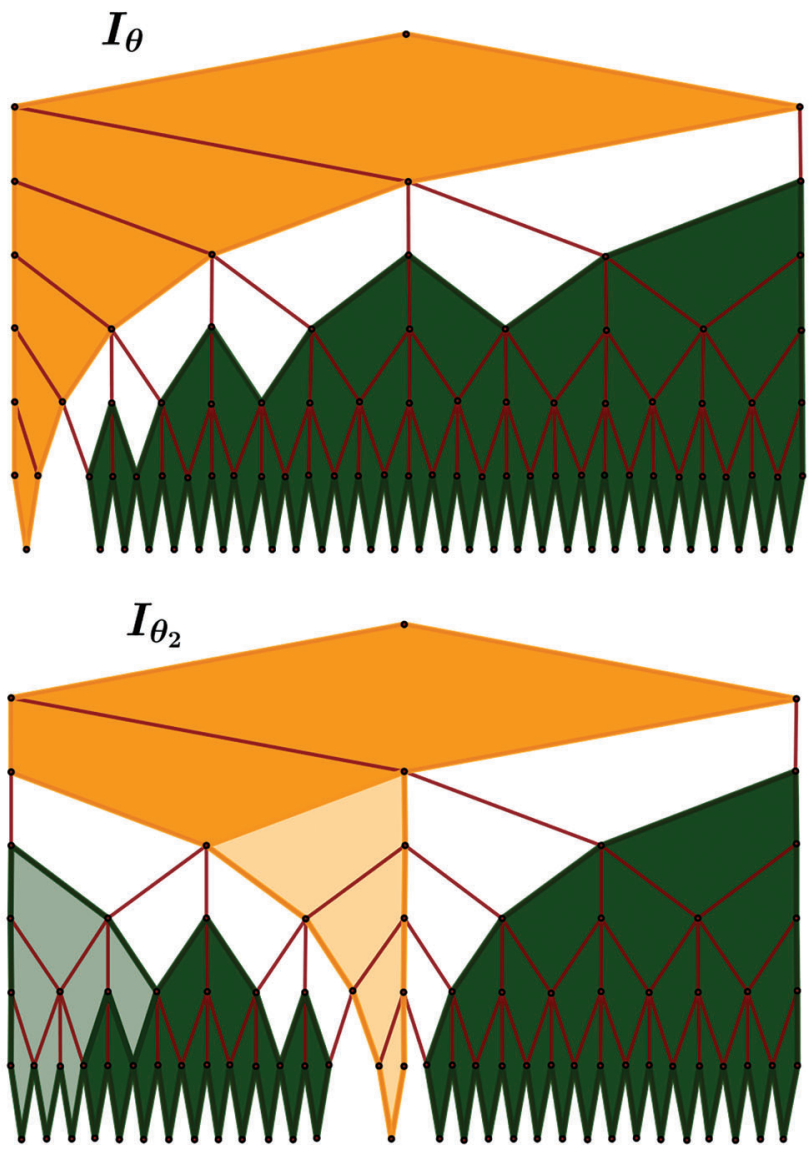

Figure 4. Ideals and their irrationals.

In Figure 4, focusing on the gold tips at the bottom of both graphs, the ideal $I_{\theta}$ determines an irrational $\theta$ that's relatively close to 0 , whereas the ideal $I_{\theta_{2}}$ determines an irrational $\theta_{2}$ that's relatively close to $1 / 2$.

Before we explain the shading, we discuss the Fell topology on the ideal space of a $\mathrm{C}^{*}$-algebra. This topology is built using representation theory of $\mathrm{C}^{*}$-algebras and establishes that the quotients move continuously in some sense with respect to ideals using a notion of operator fields [Fel61]. We will now present criteria for sequence 
convergence in this topology (the statement is also true for net convergence) along with an intriguing relationship between the Fell topology and the Hausdorff distance on a compact metric space.

Theorem 3.12 ([Fel61]). Let $A$ be a $C^{*}$-algebra. The Fell topology on Ideals $(A)$ is compact Hausdorff.

Furthermore, let $\left(I_{n}\right)_{n \in \mathbb{N}}$ be a sequence in $\operatorname{Ideals}(A)$ and $I \in$ Ideals $(A)$. The following are equivalent:

1. $\left(I_{n}\right)_{n \in \mathbb{N}}$ in $\operatorname{Ideals}(A)$ converges to $I \in \operatorname{Ideals}(A)$ with respect to the Fell topology;

2. for each $a \in A$, the sequence $\left(\left\|a+I_{n}\right\|_{A / I_{n}}\right)_{n \in \mathbb{N}}$ in $\mathbb{R}$ converges to $\|a+I\|_{A / I} \in \mathbb{R}$ with respect to the usual metric on $\mathbb{R}$.

Moreover, let $(X, d)$ be a compact metric space. If $\left(F_{n}\right)_{n \in \mathbb{N}}$ is a sequence of closed sets in $X$ and $F \subseteq X$ is closed, then using Example 3.11, the following are equivalent:

1. the sequence $\left(I_{F_{n}}\right)_{n \in \mathbb{N}}$ in $\operatorname{Ideals}(C(X))$ converges to $I_{F} \in \operatorname{Ideals}(C(X))$ with respect to the Fell topology;

2. $\left(F_{n}\right)_{n \in \mathbb{N}}$ converges to $F$ with respect to Haus $_{d}$.

This theorem shows that there is a relationship between the convergence of ideals in the Fell topology and the quotient structure regardless of the given $\mathrm{C}^{*}$-algebra. Now, for commutative $\mathrm{C}^{*}$-algebras, the next theorem shows that the Fell topology is well-suited to establish convergence of quotients in propinquity.

Theorem 3.13. Let $(X, d)$ be a compact metric space. Let $I \in$ Ideals $(C(X))$. Consider the compact set $F_{I}=\{x \in X \mid f(x)=$ $0 \forall f \in I\}$, and let $\pi_{I}: C(X) / I \rightarrow C\left(F_{I}\right)$ be the ${ }^{*}$-isomorphism given by restriction. Now, let $L_{d, I}:=L_{d} \circ \pi_{I}$, which is a $(1,0)$ Leibniz Lip-norm on $C(X) / I$.

If $\left(I_{n}\right)_{n \in \mathbb{N}}$ is a sequence in $\operatorname{Ideals}(C(X))$ that converges to $I \in \operatorname{Ideals}(C(X))$ in the Fell topology, then $\left(C(X) / I_{n}, L_{d, I_{n}}\right)_{n \in \mathbb{N}}$ converges to $\left(C(X) / I, L_{d, I}\right)$ in the Gromov-Hausdorff propinquity.

Proof. Let $I, J \in \operatorname{Ideals}(C(X))$. By the triangle inequality and equations (2) and (3), we have

$$
\begin{aligned}
& \Lambda_{(1,0)}^{*}\left(\left(C(X) / I, L_{d, I}\right),\left(C(X) / J, L_{d, J}\right)\right) \\
& \leq \Lambda_{(1,0)}^{*}\left(\left(C(X) / I, L_{d, I}\right),\left(C\left(F_{I}\right), L_{d}\right)\right) \\
&+\Lambda_{(1,0)}^{*}\left(\left(C\left(F_{I}\right), L_{d}\right),\left(C\left(F_{J}\right), L_{d}\right)\right) \\
&+\Lambda_{(1,0)}^{*}\left(\left(C\left(F_{J}\right), L_{d}\right),\left(C(X) / J, L_{d, J}\right)\right) \\
&= 0+\Lambda_{(1,0)}^{*}\left(\left(C\left(F_{I}\right), L_{d}\right),\left(C\left(F_{J}\right), L_{d}\right)\right)+0 \\
& \leq \operatorname{GH}\left(\left(F_{I}, d\right),\left(F_{J}, d\right)\right) \leq \operatorname{Haus}_{d}\left(F_{I}, F_{J}\right) .
\end{aligned}
$$

The rest of the proof follows from Theorem 3.12.

However, convergence of quotients of AF-algebras in propinquity requires more care. Theorem 3.5 and Theorem 3.8 show that the quantum metric structure on AFalgebras given by Theorem 3.5 is built from and is sensitive to the inductive limit structure. Hence, it will prove useful to form a topology on ideal spaces of inductive limits of $\mathrm{C}^{*}$-algebras that is built from the inductive limit structure. This is done in Theorem 3.14 by realizing that the inductive limit structure of the $\mathrm{C}^{*}$-algebras yields an inverse limit topology on the ideal space of the inductive limit. Moreover, in the AF case, this inverse limit recovers the Fell topology and can be given by a metric. We also show that convergence of the ideals $I_{\theta}$ in the Fell topology is the same as convergence of $\theta$ in the usual topology on $\mathbb{R}$, which in turn shows that continuity of the $\mathfrak{F} / I_{\theta}$ can be established by either $\theta$ or $I_{\theta}$.

Theorem 3.14 ([Agu19]). Let $A$ be an inductive limit of a sequence of $C^{*}$-algebras $U=\left(A_{n}\right)_{n \in \mathbb{N}_{0}}$. For each $n \in \mathbb{N}_{0}$, the map

$$
\alpha_{n}: I \in \operatorname{Ideals}\left(A_{n+1}\right) \mapsto I \cap A_{n} \in \operatorname{Ideals}\left(A_{n}\right)
$$

is continuous with respect to the Fell topologies. Thus, (Ideals $\left(A_{n}\right)$, Fell, $\left.\alpha_{n}\right)_{n \in \mathbb{N}_{0}}$ is an inverse sequence of topological spaces with inverse limit Ideals $(A)_{\infty}$ with topology Fell ${ }_{\infty}$.

If $\mathrm{Fell}_{i(U)}$ is the initial topology on $\operatorname{Ideals}(A)$ induced by $\mathrm{Fell}_{\infty}$ and the injection

$$
i(U): I \in \operatorname{Ideals}(A) \mapsto\left(I \cap A_{n}\right)_{n \in \mathbb{N}_{0}} \in \operatorname{Ideals}(A)_{\infty},
$$

then $\mathrm{Fell}_{i(U)}$ is Hausdorff and finer than the Fell topology. Furthermore, if $A$ is $A F$ and $\operatorname{dim}\left(A_{n}\right)<\infty$ for each $n \in \mathbb{N}_{0}$, then $\mathrm{Fell}_{i(U)}$ is the Fell topology and is metrized by the metric defined for each $I, J \in \operatorname{Ideals}(A)$ by

$$
m_{i(U)}(I, J)= \begin{cases}0, & I=J, \\ 2^{-\min \left\{n \mid I \cap A_{n} \neq J \cap A_{n}\right\}}, & \text { otherwise. }\end{cases}
$$

Finally, a sequence of irrationals $\left(\theta_{n}\right)_{n \in \mathbb{N}_{0}}$ in $(0,1)$ converges to an irrational $\theta \in(0,1)$ in the usual metric on $\mathbb{R}$ if and only if the sequence of ideals $\left(I_{\theta_{n}}\right)_{n \in \mathbb{N}_{0}}$ converges to $I_{\theta}$ in the metric $m_{i(U)}$ or Fell topology on $\operatorname{Ideals}(\mathfrak{F})$.

Returning to the opening graphic, the light shading is meant to reflect how far each ideal $I_{\theta_{0}}, I_{\theta_{1}}, I_{\theta_{2}}, I_{\theta_{3}}, I_{\theta_{4}}$ is from the ideal $I_{\theta}$ in the metric $m_{i(U)}$ (we only label the ideals, which are in green). For example, in Figure 4, the darker shading in the image for $I_{\theta_{2}}$ are the regions that agree with the gold and green in the image for $I_{\theta}$. Now, the more light shading there is, the further away $I_{\theta_{j}}$ is from $I_{\theta}$ in $m_{i(U)}$. Thus, the opening image is meant to elicit a sense of convergence of ideals (from the bottom left to the top right in a counterclockwise direction), and to suggest a convergence of the quotients in gold. The image also represents how convergence in $m_{i(U)}$ provides convergence of the associated irrationals since the metric is forcing the irrationals to lie in the same smaller and smaller intervals, so $\theta_{j} \rightarrow \theta$ in the usual metric on $\mathbb{R}$.

Now that we have introduced ideals and the Fell topology, we are ready to approach continuity of $\left(\mathfrak{F} / I_{\theta}\right)_{\theta \in[0,1) \backslash \mathbb{Q}}$. We will not go through the entire proof of this, but we 
will present a strategy that is commonly used for proving convergence of infinite-dimensional quantum metric spaces and was used successfully for continuity of the infinite-dimensional family $\left(\mathfrak{F} / I_{\theta}\right)_{\theta \in[0,1) \backslash \mathbb{Q}}$. It is an incredibly useful strategy to first find finite-dimensional approximations in Gromov-Hausdorff propinquity for infinitedimensional spaces to establish convergence of infinitedimensional spaces. This was done for the rotation algebras using the fuzzy tori [Lat16a, Lat15, Lat16b]. This strategy was first introduced by Rieffel near the inception of quantum metric spaces in [Rie04]. The advantage of this is that it is much easier to prove continuity results on finite-dimensional spaces (see the proof of Proposition 11.1 in [Rie04], which used the finite-dimensional properties that the unit ball is compact and that every subspace is complemented). Thus, once one has finitedimensional approximations and continuity of the finitedimensional spaces, then an application of the triangle inequality gives continuity. To see this, assume that $(A, L)$ and $\left(B, L^{\prime}\right)$ are (possibly infinite-dimensional) compact quantum metric spaces. Now, assume $\left(A_{F D}, L_{F D}\right)$ and $\left(B_{F D}, L_{F D}^{\prime}\right)$ are finite-dimensional compact quantum metric spaces that are close in Gromov-Hausdorff propinquity (the finite-dimensional approximations). We have by the triangle inequality

$$
\begin{aligned}
& \Lambda^{*}\left((A, L),\left(B, L^{\prime}\right)\right) \leq \Lambda^{*}\left((A, L),\left(A_{F D}, L_{F D}\right)\right) \\
& \quad+\Lambda^{*}\left(\left(A_{F D}, L_{F D}\right),\left(B_{F D}, L_{F D}^{\prime}\right)\right) \\
& \quad+\Lambda^{*}\left(\left(B_{F D}, L_{F D}^{\prime}\right),\left(B, L^{\prime}\right)\right)
\end{aligned}
$$

Hence, if the finite-dimensional spaces $\left(A_{F D}, L_{F D}\right)$ and $\left(B_{F D}, L_{F D}^{\prime}\right)$ are found so that they are close in propinquity (in some uniform way) to $(A, L)$ and $\left(B, L^{\prime}\right)$, respectively (which is, again, much easier than trying to establish estimates between the infinite-dimensional spaces directly), then we will have a nice estimate for $\Lambda^{*}\left((A, L),\left(B, L^{\prime}\right)\right)$.

Therefore, we should find finite-dimensional approximations for our infinite-dimensional family $\left(\mathfrak{F} / I_{\theta}\right)_{\theta \in[0,1) \backslash \mathbb{Q}}$. However, as each $\mathfrak{F} / I_{\theta}$ is $\mathrm{AF}$, we have obvious choices for finite-dimensional approximations given by inductive sequences of finite-dimensional $\mathrm{C}^{*}$-subalgebras and Theorem 3.5. Thus, for each irrational $\theta \in(0,1)$, we define a Lip-norm on $\mathfrak{F} / I_{\theta}$ by: the sequence of subalgebras $U_{\theta}=$ $\left(\left(\mathfrak{\mho}_{n}+I_{\theta}\right) / I_{\theta}\right)_{n \in \mathbb{N}_{0}}$, which is an inductive sequence of finitedimensional $C^{*}$-subalgebras for $\mathfrak{F} / I_{\theta}$; the unique faithful tracial state $\tau_{\theta}$ on $\mathfrak{F} / I_{\theta} ;$ and the sequence $\left(\beta_{\theta}(n)\right)_{n \in \mathbb{N}_{0}}=$ $\left(1 / \operatorname{dim}\left(\left(\mathfrak{F}_{n}+I_{\theta}\right) / I_{\theta}\right)\right)_{n \in \mathbb{N}_{0}}$.

Theorem 3.15 ([Agu19]). It holds that the map

$$
I_{\theta} \in \operatorname{Ideals}(\mathfrak{F}) \mapsto\left(\mathfrak{F} / I_{\theta}, L_{U_{\theta}, \tau_{\theta}}^{\beta_{\theta}}\right)
$$

is continuous with respect to the Fell topology and the GromovHausdorff propinquity. In particular, the map

$$
\theta \in(0,1) \backslash \mathbb{Q} \mapsto\left(\mathfrak{F} / I_{\theta}, L_{U_{\theta}, \tau_{\theta}}^{\beta_{\theta}}\right)
$$

is continuous with respect to the usual topology on $\mathbb{R}$ and the Gromov-Hausdorff propinquity.

We note that the metric $m_{i(U)}$ of Theorem 3.14 was crucial in obtaining continuity with respect to $\theta$ since establishing continuity of quotients with respect to ideals was a much more natural first step given the above results.

\section{Future Work}

There are still many more quantum metric properties of AFalgebras that one can look into. AF-algebras are famously classified using their so-called $K_{0}$ group, which is built using equivalence classes of projections, appropriately defined, and can be described using finitely generated projective modules. What is the relation between metric geometry and the $K_{0}$ group? For instance, the third author devised a generalization of the propinquity to a certain type of modules over quantum metric spaces (generalizing Hermitian vector bundles over manifolds), which may prove useful for finding such a relation.

More generally, the third author has introduced other generalizations of the propinquity, to make sense of the convergence of locally compact monoid actions [Lat20], or of spectral triples [Con94]. As AF-algebras are tightly connected with problems from dynamics, specifically with minimal actions on Cantor sets, the connection between the metric properties of AF-algebras and their actions with a metric picture of dynamical system could prove very interesting. The interplay between dynamics, metric geometry, and compact quantum metric spaces is a subject for current and future investigation (for some important recent work in this direction see [KK21]). Spectral triples, as a means to define a noncommutative Riemannian structure, are tools of central importance in the study of noncommutative geometry, and their metric properties are yet to be fully understood, though hopefully future work based upon the subject we brushed upon in this article will prove enlightening. Some of these new types of convergence may prove interesting even when working with commutative $\mathrm{C}^{*}$-algebras (that is, working with classical geometry) as they offer a new, functional analytic perspective on these topics.

In a different direction, the first author has begun the study of the metric properties of classes of compact quantum metric spaces related to either AF-algebras (such as bundles of AF-algebras over compact metric spaces) or to inductive limit constructions (such as $\mathrm{AT}$-algebras, which are inductive limits of algebras of matrix-valued continuous functions over the circle, and play an important role in the classification program [EE93]). In fact, the irrational

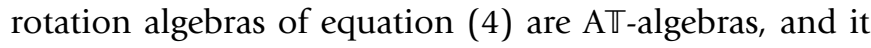
would be of interest to see if one can build a quantum metric structure on these rotation algebras from the inductive 
limit structure rather than the aforementioned structure in the first section.

Next, the propinquity is complete, as are many of its generalizations mentioned above. Thus, it is possible to discuss the construction of new $\mathrm{C}^{*}$-algebras, or new quantum metrics on known $\mathrm{C}^{*}$-algebras, using metric means. This idea could find applications in physics, and was used by the first author to construct quantum metrics on more general AF-algebras, as well as by the first and third authors and T. Rainone to build quantum metrics on BunceDeddens algebras. The completeness of the propinquity also suggests that some $\mathrm{C}^{*}$-algebras may be quantum "fractals," as attractors of some quantum iterated function system.

Finally, the topology $\mathrm{Fell}_{i(U)}$ of Theorem 3.14 bestows a topology on the ideal space of any inductive limit of $\mathrm{C}^{*}$-algebras (not just AF-algebras) that is finer than the Fell topology. Thus, it would be interesting to see when $\mathrm{Fell}_{i(U)}$ agrees with the Fell topology and to see if we can find more relationships between $\mathrm{Fell}_{i(U)}$ and classic topologies as done with the metric $m_{i(U)}$ and the irrationals (displayed by the opening image). Furthermore, Theorem 3.15 and now also Theorem 3.13 establish a fascinating connection of the Fell topology and propinquity, and motivate the search to find more examples of continuity of quotients in propinquity provided by their ideals in $\mathrm{Fell}_{i(U)}$ or the Fell topology.

ACKNOWLEDGMENTS. The first author is grateful to IMADA at Syddansk Universitet, where some of this work was completed, and gratefully acknowledges the financial support from the Independent Research Fund Denmark through the project "Classical and Quantum Distances" (grant no. 9040-00107B).

\section{References}

[AB19] Konrad Aguilar and Samantha Brooker, Quantum metrics from traces on full matrix algebras, Involve 12 (2019), no. 2, 329-342, DOI 10.2140/involve.2019.12.329. MR3864221

[Agu19] Konrad Aguilar, Fell topologies for AF-algebras and the quantum propinquity, J. Operator Theory 82 (2019), no. 2, 469-514, DOI 10.7900/jot. MR4015960

[AL15] Konrad Aguilar and Frédéric Latrémolière, Quantum ultrametrics on AF algebras and the Gromov-Hausdorff propinquity, Studia Math. 231 (2015), no. 2, 149-193, DOI 10.4064/sm227-2-5. MR3465284

[AL19] Konrad Aguilar and Alejandra López, A quantum metric on the Cantor space, arXiv:1907.05835, 2019.

[Boc08] Florin P. Boca, An AF algebra associated with the Farey tessellation, Canad. J. Math. 60 (2008), no. 5, 975-1000, DOI 10.4153/CJM-2008-043-1. MR2442043

[Con94] Alain Connes, Noncommutative geometry, Academic Press, Inc., San Diego, CA, 1994. MR1303779
[Dav96] Kenneth R. Davidson, $C^{*}$-algebras by example, Fields Institute Monographs, vol. 6, American Mathematical Society, Providence, RI, 1996, DOI $10.1090 / \mathrm{fim} / 006$. MR1402012

[EE93] George A. Elliott and David E. Evans, The structure of the irrational rotation $C^{*}$-algebra, Ann. of Math. (2) 138 (1993), no. 3, 477-501, DOI 10.2307/2946553. MR1247990

[Fel61] J. M. G. Fell, The structure of algebras of operator fields, Acta Math. 106 (1961), 233-280. MR164248

[Gro81] Mikhael Gromov, Structures métriques pour les variétés riemanniennes (French), Textes Mathématiques [Mathematical Texts], vol. 1, CEDIC, Paris, 1981. Edited by J. Lafontaine and P. Pansu. MR682063

[JRZ18] Marius Junge, Sepideh Rezvani, and Qiang Zeng, Harmonic analysis approach to Gromov-Hausdorff convergence for noncommutative tori, Comm. Math. Phys. 358 (2018), no. 3, 919-994, DOI 10.1007/s00220-017-3017-4. MR3778347

[KK21] Jens Kaad and David Kyed, Dynamics of compact quantum metric spaces, Ergodic Theory Dynam. Systems 41 (2021), no. 7, 2069-2109, DOI 10.1017/etds.2020.34. MR4266364

[KL09] David Kerr and Hanfeng Li, On Gromov-Hausdorff convergence for operator metric spaces, J. Operator Theory 62 (2009), no. 1, 83-109. MR2520541

[Lat15] Frédéric Latrémolière, The dual Gromov-Hausdorff propinquity, J. Math. Pures Appl. (9) 103 (2015), no. 2, 303351. MR3298361

[Lat16a] Frédéric Latrémolière, The quantum GromovHausdorff propinquity, Trans. Amer. Math. Soc. 368 (2016), no. 1, 365-411, DOI 10.1090/tran/6334. MR3413867

[Lat16b] Frédéric Latrémolière, Quantum metric spaces and the Gromov-Hausdorff propinquity, Noncommutative geometry and optimal transport, Contemp. Math., vol. 676, Amer. Math. Soc., Providence, RI, 2016, pp. 47-133. MR3578737

[Lat20] Frédéric Latrémolière, The covariant Gromov-Hausdorff propinquity, Studia Math. 251 (2020), no. 2, 135-169. MR4045657

[Rie04] Marc A. Rieffel, Gromov-Hausdorff distance for quantum metric spaces. Matrix algebras converge to the sphere for quantum Gromov-Hausdorff distance, Mem. Amer. Math. Soc. 168 (2004), no. 796, 1-65, DOI $10.1090 / \mathrm{memo} / 0796$. Appendix 1 by Hanfeng Li. MR2055927

[Rie16] Marc A. Rieffel, Matricial bridges for "matrix algebras converge to the sphere", Operator algebras and their applications, Contemp. Math., vol. 671, Amer. Math. Soc., Providence, RI, 2016, pp. 209-233, DOI 10.1090/conm/671/13512 MR3546687

[Rie98] Marc A. Rieffel, Metrics on states from actions of compact groups, Doc. Math. 3 (1998), 215-229. MR1647515 


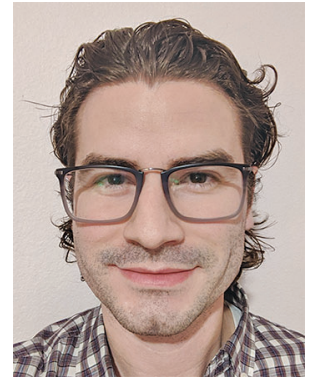

Konrad Aguilar

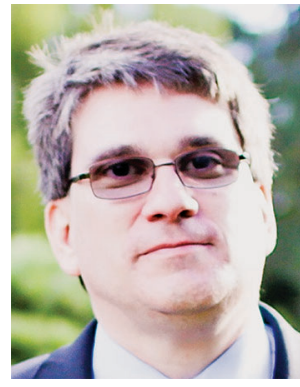

Frédéric

Latrémolière

\section{Credits}

All figures are courtesy of the authors and were drawn in $\mathrm{Ge}$ ometry by GeoGebra. The design of the opening graphic and Figure 4 was influenced by the images created by F. P. Boca in [Boc08].

Photo of Konrad Aguilar is courtesy of Konrad Aguilar.

Photo of Samantha Brooker is courtesy of Kimberly Fitchmun.

Photo of Frédéric Latrémolière is courtesy of Frédéric Latrémolière.

Photo of Alejandra López is courtesy of Alejandra López.

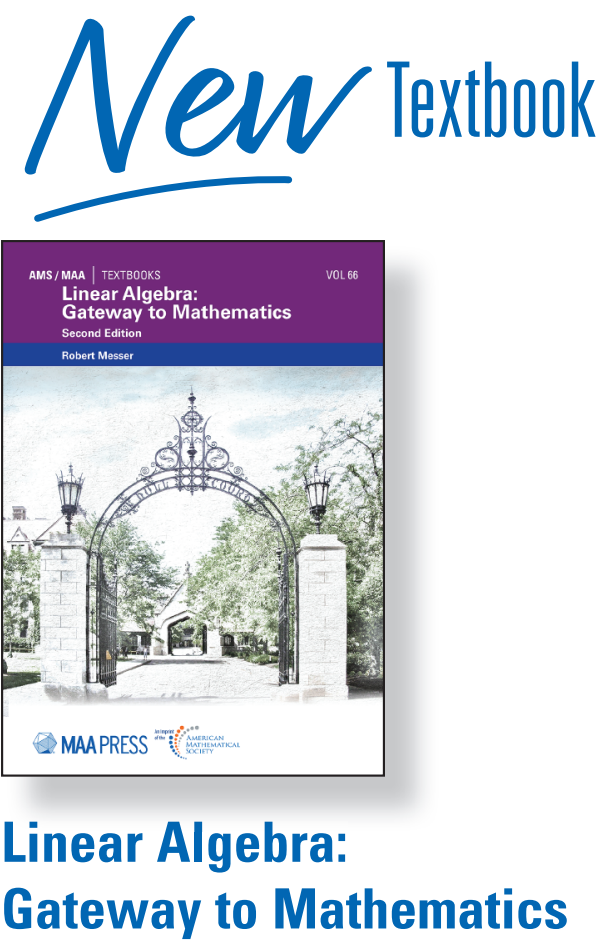

\section{Second Edition}

Robert Messer, Albion College, MI

Linear Algebra: Gateway to Mathematics uses linear algebra as a vehicle to introduce students to the inner workings of mathematics. The structures and techniques of mathematics in turn provide an accessible framework to illustrate the powerful and beautiful results about vector spaces and linear transformations.

The unifying concepts of linear algebra reveal the analogies among three primary examples: Euclidean spaces, function spaces, and collections of matrices. Students are gently introduced to abstractions of higher mathematics through discussions of the logical structure of proofs, the need to translate terminology into notation, and efficient ways to discover and present proofs.

AMS/MAA Textbooks, Volume 66; 2021; 434 pages; Softcover; ISBN: 978-1-4704-6295-6; List US\$89; AMS members US\$66.75; MAA members US\$66.75; Order code TEXT/66

\section{$=$ Textbook \\ Visit bookstore.ams.org}

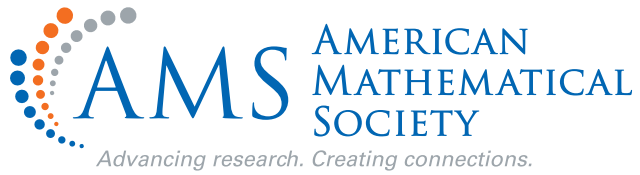

